\title{
Pregabalin Addiction: Case Report of a Young Adult
}

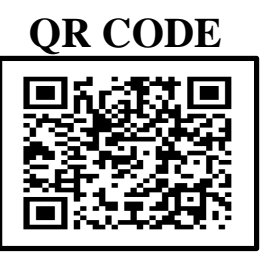

\section{NITIN CHAUDHARY*1, SAPNA JAIN²}

Pregabalin has been used widely for the treatment of neuropathic pain, generalized anxiety disorders and epilepsy. Data is scarce and much is not known about the addictive potential of pregabalin. Herein, we report details of a young adult who got addicted to pregabalin and approached for a smooth withdrawal. The subject had a successful outcome. Literature suggests a growing concern for the abuse of pregabalin. Risk factors associated with the development of addictive behavior with pregabalin need to be assessed.

KEYWORDS: Pregabalin, Addiction, Abuse, Dependence

\section{INTRODUCTION}

Pregabalin is a drug that exerts anticonvulsant, analgesic and anxiolytic effects on animal models and has a high affinity for the voltage-dependent calcium channels. ${ }^{1}$ It decreases the influx of calcium induced by depolarization and thus reduces the emissions of numerous excitatory. ${ }^{1}$ The prescribing indications include fibromyalgia, post herpetic neuralgia and neuropathic pain after spinal cord injury or due to diabetes mellitus. ${ }^{2}$ It is not approved for generalized anxiety disorders ${ }^{3}$ but is often used off-labelly for psychiatric and addictive disorders such as insomnia, obsessive-compulsive disorder, post-traumatic stress syndrome, anxiety in schizophrenia, the treatment of benzodiazepine withdrawal and dependence as well as the prevention of relapse in alcohol dependence. ${ }^{4-11}$ It has also been shown the benefit of pregabalin in reducing the opioid abstinence syndrome. ${ }^{12,13}$

In 2010, on the basis of data analyzed from the Swedish national adverse drug reactions register, it was concluded that pregabalin was likely to be associated with a potential for abuse. ${ }^{14}$ Despite the increasing evidence of abuse, pregabalin is increasingly being prescribed. A 2012 report in the United Kingdom noted that the prescribing of pregabalin had increased by $350 \%$ over the previous five years ${ }^{15}$ The potential risk of abuse and addiction, and of the risks of liver and hematological toxicity were published in $2012^{16}$ and recently in 2019, another manuscript warned about the risk of pregabalinrelated suicide. ${ }^{17}$ As pregabalin is being studied as a treatment in addiction, especially for the treatment of benzodiazepine withdrawal ${ }^{10}$ and for the prevention of relapse in alcohol dependence ${ }^{\mathrm{Il}}$, assessment of its potential for addiction is of major interest. As pregabalin has been considered to have a low potential for abuse, many healthcare professionals don't take this product-use issue much into account when prescribing. However, several cases have been published and a few professionals are concerned about the risks and limitations of prescribing this drug. This facilitates the early detection of problematic developments before they become a major problem. Other data are necessary in order to identify risk factors for pregabalin abuse and dependence to optimize its medical indications and prescribing practices. We present a case of a young adult who presented himself with a request of pregabalin withdrawal.

\section{CASE REPORT}

A 25 year old male working as a sales representative with a telecom company visited us after being referred from his company panel doctor for pregabalin withdrawal. He had been taking between $1.8 \mathrm{~g}$ of pregabalin daily for a year with no other associated drug use. He had tried to stop the drug few times, but withdrawal symptoms appeared every time. On further questioning he detailed that the symptoms included sweating, tremors, diarrhea, asthenia, joint pain and a craving to start pregabalin again, had appeared each time. His history included Marijuana abuse and opiate misuse that started during high college days till he started working at the age of 23. He also had a history of hospitalization due to alcohol dependence multiple times during college 
days. He claimed to have tried a few unknown drugs by injection. He had only tried buprenorphine had been abstinent from opiates since. He had no other somatic or psychiatric history and there was no history of addiction or psychiatric disorder in his family. He started taking pregabalin after he was suggested by a college senior regarding its potential for helping in management of Marijuana and opioid withdrawal. He started taking pregabalin $300 \mathrm{mg}$ once in a day. This helped him to decrease using marijuana but after a few months he increased the dose to twice daily as he wanted to quit marijuana completely. Gradually he increased the dose to 6 tablets in 3 divided doses per day.

The sought-after effects were calmness, improved sociability, a feeling of well-being and relaxation and an improvement in sleep. We hospitalized him and gradually reduced the doses, decreasing by $100 \mathrm{mg}$ per day in the with daily dispensing. During hospitalization, the patient had a very strong desire to use pregabalin and requested treatment to help him; he also complained of gastrointestinal problems, insomnia, muscle and joint pain, sweating and anxiety. Amisulpride and chlorpromazine were started. Gradually decreasing benzodiazepine treatment was introduced for the craving. He sometimes showed a desire for a single pregabalin dose. High dosage buprenorphine substitution treatment was introduced. His condition got much better and he was discharged. The withdrawal symptoms were no longer present at the time of discharge. He was stable for several months before losing for follow up.

\section{DISCUSSION}

Abuse and dependence of many drugs has come up in the past few decades. The euphoric effect of pregabalin can be considered as the main reason behind its abuse. Experiencing euphoria appears to be a dose-dependent side effect of pregabalin which encourages some patients to ingest large doses and appears to be a transient side effect. Supratherapeutic doses may produce sedation, dissociation, numbness, disinhibition, improved sociability, and auditory and visual hallucinations. ${ }^{18,19}$ In our case, pregabalin was initiated with the aim of coming off another substance, but the initial withdrawal ultimately turned into a new dependence. The subject succeeded in withdrawing from pregabalin but required management based on the prescribing of opioid substitution treatment.
Pregabalin abuse typically involves supra-therapeutic doses, often clearly exceeding the maximum recommended dose of $600 \mathrm{mg}$ administered in divided doses. Abusers may continue to increase the dose because tachyphylaxis rapidly develops. ${ }^{18}$ Although most take these drugs orally, other routes of administration have been reported: injection, smoking or inhalation of crushed tablets, rectal or parachuting. ${ }^{20,21}$ There are more cases of pregabalin dependence than of abuse.

Most of the patients developed withdrawal symptoms on stopping pregabalin. Of the disorders related to substance use, the use of opiates is the most significant risk factor. In Germany, $12.1 \%$ of the patients treated for opioid dependence were abusing pregabalin compared to $2.7 \%$ of the patients treated for addictions to substances other than opiates. ${ }^{22}$ Similarly, in a population of former prisoners, those with disorders related to opioid use were more likely to abuse pregabalin $(26 \%)$ than those with a disorder related to the use of non-opioid substances $(4 \%)$. $^{23-26}$ In most cases, pregabalin is initially been prescribed to treat a medical condition, which suggests that the development of addictive behaviors associated with pregabalin may also occur in the context of normal medical treatment. Moreover, it is being used for many unapproved indications.

The management of the patients varies greatly from one patient to another, hospitalization and associated treatment may or may not be required. There is no consensus on the therapeutic management of pregabalin withdrawal. There has clearly been an increase in the frequency of cases of pregabalin abuse or dependence in recent years. ${ }^{23,27}$ This observation probably relates to both an increase in reports, given the increased awareness of pregabalin's potential for addiction, and a real increase in the incidence of pregabalin abuse or dependence. Drug abuse or dependence may increase for some time before it reaches the threshold for reporting.

\section{CONCLUSION}

Pregabalin addiction may have reached the clinical detection threshold and the request for treatment is likewise increasing. There is a need to explore about certain characteristics of pregabalin misuse and addiction. The pharmacokinetic aspects of pregabalin need to be considered when assessing its potential for dependence. The current literature suggests an increase in pregabalin abuse. There is need for a 
treatment specifically for problems associated with pregabalin use. The case presented in this article suggests that pregabalin should be prescribed with caution in patients with a history of addiction, especially to opiates. This potential for abuse is even more significant as pregabalin is being used as a treatment of another drug addiction. Large sample studies are required to assess the Risk factors associated with the development of addictive behavior with pregabalin. There is also a need to propose an appropriate management for the same.

\section{REFERENCES}

1. Martinotti G, Lupi M, Sarchione F, et al. The potential of pregabalin in neurology, psychiatry and addiction: a qualitative overview. Curr Pharm Des 2013;19(35):6367-74.

2. Pexton T, Moeller-Bertram T, Schilling JM, et al. Targeting voltage-gated calcium channels for the treatment of neuropathic pain: a review of drug development. Expert Opin Investig Drugs 2011;20(9):1277-84.

3. Wettermark B, Brandt L, Kieler H, et al. Pregabalin is increasingly prescribed for neuropathic pain, generalised anxiety disorder and epilepsy but many patients discontinue treatment. Int J Clin Pract 2014;68(1):104-10.

4. Cho YW, Song ML. Effects of pregabalin in patients with hypnotic-dependent insomnia. J Clin Sleep Med 2014;10(5):545-50.

5. Di Nicola M, Tedeschi D, Martinotti G, et al. Pregabalin augmentation in treatment-resistant obsessive-compulsive disorder: a 16-week case series. J Clin Psychopharmacol 2011;31(5):675-7.

6. Strawn JR, Dowling BP, Geracioti Jr TD. Pregabalin treatment of posttraumatic stress disorder. J Clin Psychopharmacol 2008;28(5):596-7.

7. Pae CU. Pregabalin augmentation to antidepressants in patients with major depressive disorder. Prog Neuropsychopharmacol Biol Psychiatry 2009;33(3):577-8.

8. Schönfeldt-Lecuona C, Wolf RC, Osterfeld ND, et al. Pregabalin in the treatment of schizophrenic anxiety. Pharmacopsychiatry 2009;42(3):124-5.

9. Oulis P, Kalogerakou S, Anyfandi E, et al. Cognitive effects of pregabalin in the treatment of long-term benzodiazepine-use and dependence. Hum Psychopharmacol 2014;29(3):224-9.

10. Biermann T, Bleich S, Kornhuber J, et al. Pregabalin in benzodiazepine withdrawal. Pharmacopsychiatry 2007;40(6):292-3.
11. Martinotti G, Di Nicola M, Tedeschi D, et al. Efficacy and safety of pregabalin in alcohol dependence. Adv Ther 2008;25(6):6o8-18.

12. Krupitsky EM, Ilyuk RD, Mikhailov AD, et al. A randomised single blind study of the efficacy of pregabalin in the treatment of opioid withdrawal syndrome. Zh Nevrol Psikhiatr Im SS Korsakova 2016;116(7):29-36.

13. Kämmerer N, Lemenager $T$, Grosshans $M$, et al. Pregabalin for the reduction of opiate withdrawal symptoms. Psychiatr Prax 2012;39(7):351-2.

14. Schwan S, Sundström A, Stjernberg E, et al. A signal for an abuse liability for pregabalin-results from the Swedish spontaneous adverse drug reaction reporting system. Eur J Clin Pharmacol 2010;66(9):947-53.

15. Spence D. Bad medicine: gabapentin and pregabalin. BMJ 2013;347:f6747.

16. Gabapentine, prégabaline : abus et dépendances. Rev Prescrire2012;32(340):116-8.

17. Prégabaline: suicides. Rev Prescrire 2019;39(434):911.

18. Halaby A, Kassm SA, Naja WJ. Pregabalin dependence: a case report. Curr Drug Saf 2015;10(2):184-6.

19. Skopp G, Zimmer G. Pregabalin-a drug with abuse potential? Arch Kriminol 2012;229(1-2):44-54.

20. Carrus D, Schifano F. Pregabalin misuse-related issues; intake of large dosages, drug-smoking allegations, and possible association with myositis: two case reports. J Clin Psychopharmacol 2012;32:839-40.

21. Muquebil A, Al Shaban Rodríguez W, Álvarez G. Abuso de pregabalina enpaciente de perfil impulsivo: tratamiento con topiramato. Psiquiatr Biológica2016;23(2):74-6.

22. Grosshans M, Lemenager T, Vollmert C, et al. Pregabalin abuse among opiate addicted patients. Eur J Clin Pharmacol 2013;69(12):2021-5.

23. Schifano F, D'Offizi S, Piccione M, et al. Is there a recreational misuse potential for pregabalin? Analysis of anecdotal online reports in comparison with related gabapentin and clonazepam data. Psychother Psychosom 2011;80(2):118-22.

24. Schifano F. Misuse and abuse of pregabalin and gabapentin: cause for concern? CNS Drugs 2014;28:491-6.

25. Bockbrader HN, Burger P, Knapp L. Pregabalin effect on steady-state pharmaco-kinetics of carbamazepine, lamotrigine, phenobarbital, phenytoin, topiramate, valproate, and tiagabine. Epilepsia 2011;52(2):405-9. 
26. Bastiaens L, Galus J, Mazur C. Abuse of gabapentin is associated with opioid addiction. Psychiatr Q 2016;87(4):763-7.

27. Gahr M, Freudenmann RW, Hiemke $C$, et al. Pregabalin abuse and dependence in Germany: results from a database query. Eur J Clin Pharmacol 2013;69(6):1335-42.

Source of support: Nil, Conflict of interest: None declared

Cite this article as:

Chaudhary N, Jain S. Pregabalin Addiction: Case Report of a Young Adult. Int Healthc Res J. 2020;4(3):60-63. https://doi.org/10.26440/IHRJ/0403.06172

\section{AUTHOR AFFILIATIONS:}

1. MD (Medicine), Private Practitioner and Consultant Doctor, Bhopal, India

2. MBBS, Private Practitioner, Smiles Multispeciality Clinic, Bhatinda, India

Contact Corresponding Author at: chaudhary_nitin7844[at]gmail[dot]com 